\title{
SwissNET: das Schweizerische Register für Neuro-Endokrine Tumoren
}

\author{
Aurel Perren ${ }^{a}$, \\ Christoph Stettler ${ }^{b}$ \\ a Prof. Dr. med. \\ Präsident SwissNET \\ b PD Dr. med., Registerleiter
}

\begin{abstract}
Neuro-Endokrine Tumoren sind selten, und es steht eine grosse Palette von Behandlungsoptionen in verschiedenen medizinischen Fachgebieten zur Verfügung. Dies führt dazu, dass es meist wenig Evidenz für die eine oder andere Therapieoption gibt. Auch die publizierten epidemiologischen Daten sind wahrscheinlich unzuverlässig. Im Dezember 2005 wurde die interdisziplinäre SwissNET Gesellschaft gegründet. Sie vereinigt Interessierte aus verschiedenen Fachrichtungen, z.B. aus Chirurgie, Endokrinologie, Onkologie, Nuklearmedizin, Pathologie und Gastroenterologie. Das primäre Ziel ist die Führung eines nationalen Registers für Neuro-Endokrine Tumoren, wie es bereits zum Beispiel in Frankreich und Deutschland besteht.

Um möglichst lückenlos die betroffenen Patientinnen und Patienten der Schweiz erfassen zu können, möchte SwissNET, analog zu den kantonalen Krebsregistern, die Ersterfassung der betroffenen Patienten über die Pathologie-Institute sowie die behandelnden Ärzte und insbesondere auch Hausärzte vornehmen. Deshalb ist das Register auf Ihre Mitarbeit angewiesen, und der Erfolg des Registers ist von dieser Mitarbeit abhängig.
\end{abstract}

\section{Neuro-Endokrine Tumoren sind selten, und es steht eine grosse Palette von Behandlungsoptionen zur Verfügung}

Bestehende Probleme beim Aspekt des Datenschutzes konnten unter Berücksichtigung spezieller Auflagen ausgeräumt werden. Im Februar 2009 hat das BAG dem Verein SwissNET eine Sonderbewilligung zur Führung eines Registers erteilt. Ein Zitat aus der Bewilligung beschreibt deren Zweck: «Alle dem Schweizer Register für Neuro-Endokrine Tumore übermittelten Patientendaten dürfen einzig der Registrierung der in der Bewilligung umschriebenen Tumorarten die-

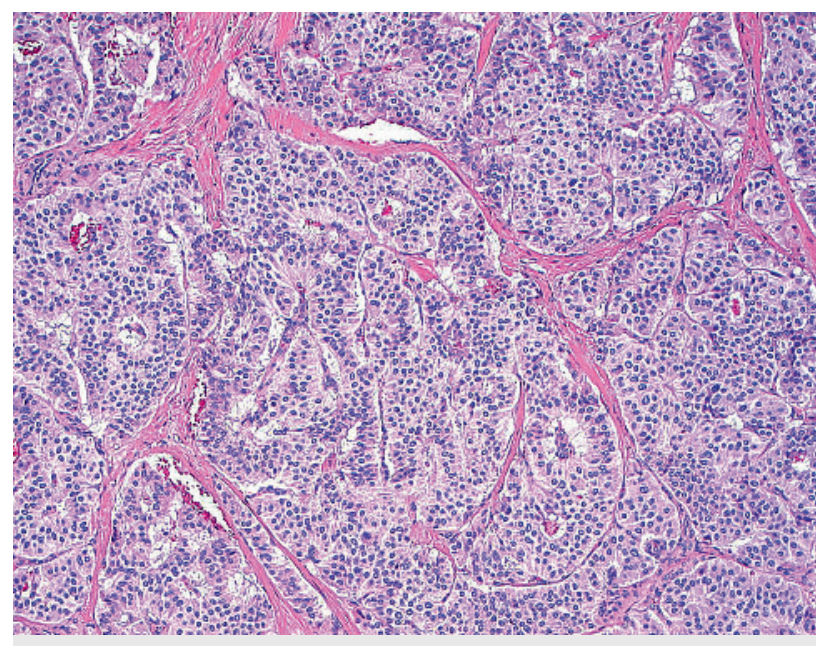

Typisches histopathologisches Bild eines endokrinen Tumors, hier eines Insulinoms des Pankreas.

Mit dieser Bewilligung wird allen in der Schweiz tätigen Ärzten, Kliniken und Instituten die Erlaubnis erteilt, dem Verein SwissNET Namen und Adressen von Patienten mit Neuro-Endokrinen Tumoren sowie den behandelnden Hausarzt direkt zu melden, um bei den Patienten eine Einwilligung zur Aufnahme ins Register einholen zu können. Die Patienten werden im Rahmen der Aufklärung über ihr Recht informiert, eine Aufnahme ins Register verweigern zu können. Falls dieses Vetorecht von den Patienten direkt bei den behandelnden Hausärzten ausgeübt wird, darf dieser keinerlei Daten an das SwissNET-Register weiterleiten.

Verzichten die Patienten auf die Ausübung ihres Vetorechts, so dürfen von den behandelnden Ärzten Daten, die in Zusammenhang mit der Erkrankung an einem Neuro-Endokrinen Tumor stehen und dem oben angeführten Zweck dienen, dem SwissNET-Register übermittelt werden. Ebenso darf Einsicht in diesbezügliche Patientenakten gewährt werden. Vollständige Krankengeschichten, Untersuchungsberichte, Befunde usw. dürfen nicht direkt übermittelt werden.

Damit das Tumorregister für Neuro-Endokrine Tumore erfolgreich geführt werden kann, sind wir auf Ihre Unterstützung angewiesen. Die bei SwissNET angestellte «Study nurse»* wird sich bei PathologieInstituten und Hausärzten melden. Für weitere Fragen stehen wir Ihnen gerne jederzeit zur Verfügung. \footnotetext{
nen. Es handelt sich um eine kontinuierliche und systematische Datenerfassung zur Analyse und Interpretation von Daten möglichst aller auf dem Gebiet der Schweiz an Neuro-Endokrinen Tumoren erkrankten Patienten. Das Register bezweckt die Bereitstellung der Grundlagen für die epidemiologische Erforschung der relativ seltenen Neuro-Endokrinen Tumore.»

niversität Be

CH-3010 Bern

Tel. 0316323222

Fax 0316324995

SwissNET

c/o Prof. Dr. med. Aurel Perren
} 